\title{
Фотореверсивный ток в светодиодных гетероструктурах на основе InGaN/GaN с разным количеством квантовых ям
}

\author{
(C) А.Э. Асланян ${ }^{1}$, Л.П. Авакянц ${ }^{1}$, А.В. Червяков ${ }^{1}$, А.Н. Туркин ${ }^{1}$, В.А. Курешов ${ }^{2}$, \\ Д.Р. Сабитов ${ }^{2}$, А.А. Мармалюк ${ }^{2}$ \\ ${ }^{1}$ Московский государственный университет им. М.В. Ломоносова (физический фракультет), \\ 119991 Москва, Россия \\ ${ }^{2} \mathrm{OOO} \mathrm{„Сигм} \mathrm{плюс“,}$ \\ 117342 Москва, Россия \\ E-mail: aslanyan.artyom@physics.msu.ru
}

Поступила в Редакцию 28 октября 2019 г.

В окончательной редакции 6 ноября 2019 г.

Принята к публикации 6 ноября 2019 г.

\begin{abstract}
Исследованы светодиодные гетероструктуры на основе InGaN/GaN с различным количеством квантовых ям методом спектроскопии фототока в диапазоне длин волн $350-500$ нм. В результате анализа серии спектров, полученных при различных смещениях $p-n$-перехода, обнаружен эффект смены направления фототока при изменении длины волны возбуждения (фотореверсивный эффект). Установлен диапазон смещений $p-n$-перехода, при котором наблюдается указанный эффект для структур с разным количеством квантовых ям в активной области.
\end{abstract}

Ключевые слова: светодиодные гетероструктуры, фототок, квантовая яма, нитрид галлия.

DOI: 10.21883/FTP.2020.03.49035.9296

\section{1. Введение}

Развитие технологии изготовления приборов на основе гетероструктур с квантовыми ямами (КЯ) InGaN/GaN невозможно без детального изучения процессов фотовозбуждения и протекания тока вдоль направления роста структуры. Такие исследования важны как для поглощающих [1], так и для светоизлучающих устройств на основе $\mathrm{GaN}$ [2], используемых в ультрафиолетовом, синем и зеленом диапазонах оптического спектра. Одним из инструментов для таких исследований является метод спектроскопии фототока (ФТ). Этот метод связан с поглощением монохроматического излучения при фотовозбуждении связанных электронов из валентной зоны в зону проводимости [3]. Ранее такие исследования проводились для структур на основе слоев InGaN/GaN с разной концентрацией индия в квантовых ямах активной области [4], при гидростатическом давлении [5], с разной концентрацией $\mathrm{Mg}$ в барьерах [6]. В указанных работах авторы изменяли состав, количество слоев, а также концентрацию примесей в активной области с целью поиска оптимальной зонной структуры готового устройства.

Помимо состава слоев и концентрации примесей, зонная структура определяется напряженностью электрического поля в этих слоях. Внешнее электрическое поле, приложенное к $p-n$-переходу с помощью источника постоянного напряжения, позволяет изменять наклон зон. В этом случае обратное напряжение приводит к уменьшению наклона зон в КЯ InGaN и увеличению в барьерах $\mathrm{GaN}$. Кроме того, увеличивается разность энергий между зонами одного типа в $n$-GaN- и $p$-GaN-областях и между барьерами активной области. Таким образом, конфигурация зонной структуры задает высоту потенциальных барьеров для носителей зарядов, оказывая влияние на величину и направление тока в структуре. В гетероструктурах с $p-n$-переходом можно изменять внутренние электрические поля по направлению и величине, подавая смещение на соответствующие контакты. Небольшое смещение вызывает существенное изменение внутренних электрических полей за счет высокоомной обедненной области, в которой расположены активные элементы структуры - квантовые ямы. Таким образом, исследованные в данной работе светодиодные гетероструктуры являются модельными объектами для изучения процессов поглощения и излучения света в полупроводниковых гетероструктурах.

В настоящей работе представлены результаты исследования спектров ФТ при смещениях $p-n$-перехода разного знака в светодиодных гетероструктурах InGaN/GaN с разным количеством КЯ в активной области.

\section{2. Образец и методика эксперимента}

Исследованные образцы были получены методом газофазной эпитаксии из металлоорганических соединений (MOCVD). Последовательно на сапфировой подложке диаметром 50.8 мм и толщиной 430 мкм выращивались затравочный низкотемпературный слой и буферный слой $i$-GaN суммарной толщиной 2 мкм; слой $n-\mathrm{GaN}$, легированный $\mathrm{Si}$ с концентрацией доноров $5 \cdot 10^{18} \mathrm{~cm}^{-3}$; активная область, состоящая из барьера $i$-GaN толщиной 16 нм и пар InGaN/GaN с толщинами 2.5/15 нм, составляющих КЯ; слой $p-\mathrm{GaN}$ толщиной 110 нм с концентрацией акцепторов $2 \cdot 10^{19} \mathrm{~cm}^{-3}$ (рис. 1). На поверхности образцов были сформированы индиевые контакты к слоям $n-\mathrm{GaN}$ и $p-\mathrm{GaN}$. Длина волны свечения 


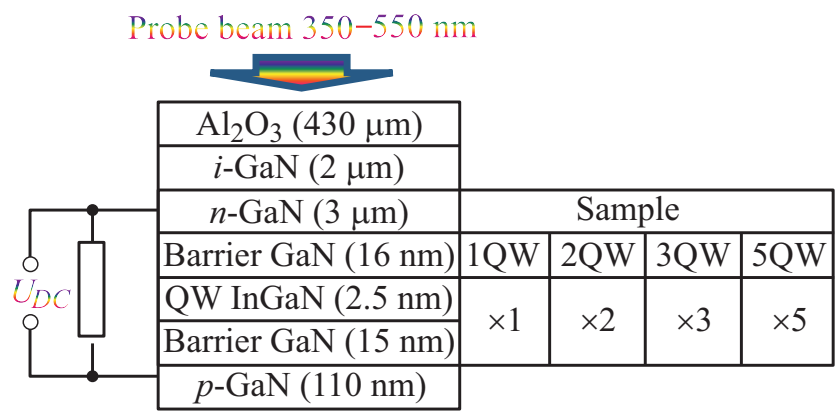

Рис. 1. Структура образца и схема подключения смещения $U_{D C}$ к $p-n$-переходу.

структур $~ 510$ нм по данным спектроскопии электролюминесценции.

В светодиодных структурах на основе InGaN/GaN, как правило, применяют специальные слои $\mathrm{AlGaN}$, ограничивающие перемещение носителей вдоль направления роста структуры. Это позволяет уменьшить утечку электронов из активной области, что важно для мини- мизации эффекта резкого уменьшения эффективности инжекции с ростом тока [7]. Однако в данной работе для уменьшения количества факторов, влияющих на ФТ и зонную структуру, были использованы образцы без слоeв AlGaN. Для регистрации спектров ФТ использовалась экспериментальная установка, реализующая метод синхронного детектирования. Для этого свет от лампы накаливания, прошедший через монохроматор в диапазоне длин волн 350-550 нм, модулировался механическим прерывателем с частотой 370 Гц. Затем модулированное излучение падало на образец под углом $8^{\circ}$. Сигнал с прерывателя (меандр) подавался на вход опорного напряжения синхронного детектора. Напряжение на контактах $p-n$-перехода пропорционально переменной составляющей тока, возникающей между $p$ - и $n$-контактами при модуляции излучения из монохроматора. Это же напряжение подавалось на вход синхронного детектора. Таким образом, напряжение на выходе синхронного детектора было пропорционально фототоку, возникающему в структуре. Постоянное смещение $p$-n-перехода $U_{D C}$ с источника напряжения изменялось в диапазоне $-1 \mathrm{~B} . .+4$ В с шагом $0.5 \mathrm{~B}$.
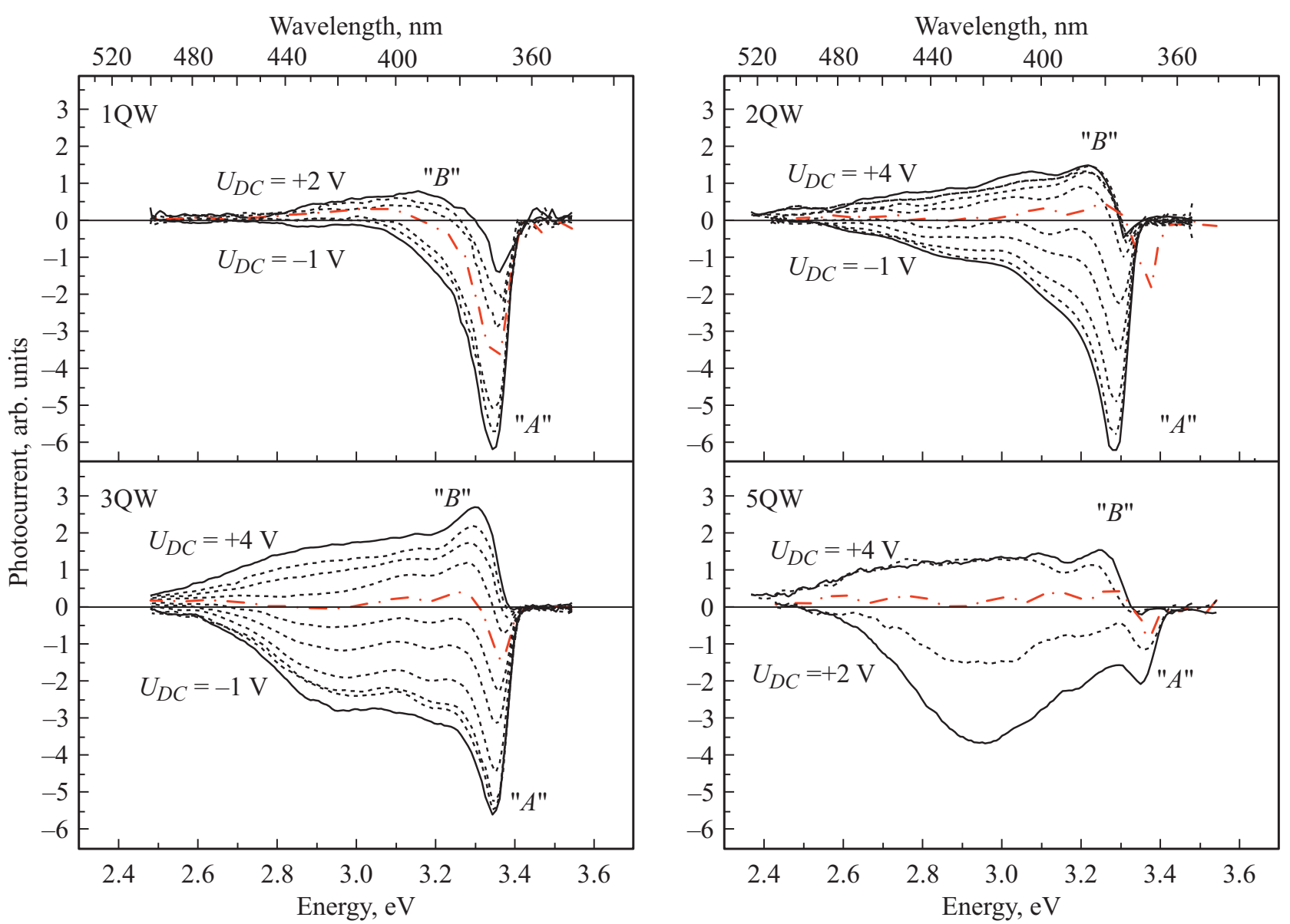

Рис. 2. Спектры ФТ при разных смещениях $p-n$-перехода $U_{D C}$ для образцов с $1,2,3$ и 5 КЯ в активной области. Сплошной линией обозначены спектры ФТ при минимальных и максимальных смещениях $U_{D C}$ в серии. Штриховой линией обозначены спектры ФТ при промежуточных смещениях $U_{D C}$. Штрихпунктирной линией выделены спектры ФТ при минимальном смещении $p-n$-перехода $U_{D C}$, при котором возникает фотореверсивный эффект. 


\section{3. Результаты и обсуждение}

Как правило, для анализа спектров ФТ проводится нормировка либо на максимальное значение фототока $[8,9]$, либо на фототок при энергии, относящейся к фундаментальному краю поглощения GaN [10]. Такая процедура позволяет определить ширину запрещенной зоны и избежать влияния смещения $p-n$-перехода $U_{D C}$ на изменения амплитуды сигнала фототока, связанного с изменением квантового выхода. В данной работе, в связи с необходимостью анализа величины фототока, нормировка не проводилась.

Спектры ФТ для образцов с 1, 2, 3 и 5 КЯ при разных смещениях $p-n$-перехода $U_{D C}$ представлены на рис. 2 . Сплошной линией выделены спектры, соответствующие максимальному положительному и отрицательному смещению $U_{D C}$. Штриховыми линиями обозначены спектры с промежуточными значениями $U_{D C}$ с шагом $0.5 \mathrm{~B}$. Для каждого образца красной штрихпунктирной линией обозначен спектр при минимальном смещении $U_{D C}$, при котором наблюдается фотореверсивный эффект. Этот эффект состоит в следующем. Обычно фототок может быть только прямым или обратным при любой длине волны возбуждения (например, сплошная линия для образца с тремя КЯ на рис. 2). Но мы обнаружили, что фототок может менять направление в зависимости от длины волны возбуждающего излучения в определенном диапазоне смещений. На рис. 2 штрихпунктирной линией выделены спектры ФТ при минимальном смещении $p-n$-перехода $U_{D C}$, при котором возникает фотореверсивный эффект. Указанный эффект может быть объяснен следующими процессами:

1) поглощением в нелегированных барьерных слоях $\mathrm{GaN}$ при энергии около $3.34-3.37$ эВ. Соответствующий спектральный пик обозначен ,А“ на рис. 2. Этот спектральный пик создает обратный ток практически при всех смещениях;

2) поглощением при участии донорно-акцепторных переходов в диапазоне энергий 2.6-3.3 эВ [11]. Соответствующий спектральный пик обозначен „B“ на рис. 2. Этот спектральный пик создает прямой ток.

В случае процесса „A“ электроны возбуждаются из валентной зоны барьера в зону проводимости (рис. 3). Свободные электроны, сформированные таким образом, создают обратный ток даже при небольшом прямом смещении $p-n$-перехода. С ростом прямого смещения электрическое поле в барьере уменьшается, зоны выравниваются и обратный ток прекращается. Наблюдается смещение пика „А“ в коротковолновую область спектра.

Поглощение в случае процесса „B“ объясняется возбуждением электрона в зону проводимости с акцепторного уровня валентной зоны барьерного слоя около области $p$-GaN. Потенциальный барьер для таких электронов достаточно мал для прямого тока, возникающего даже при отрицательном смещении. С ростом смещения прямой ток будет увеличиваться в соответствии с процессами в гомогенном $p-n$-переходе.

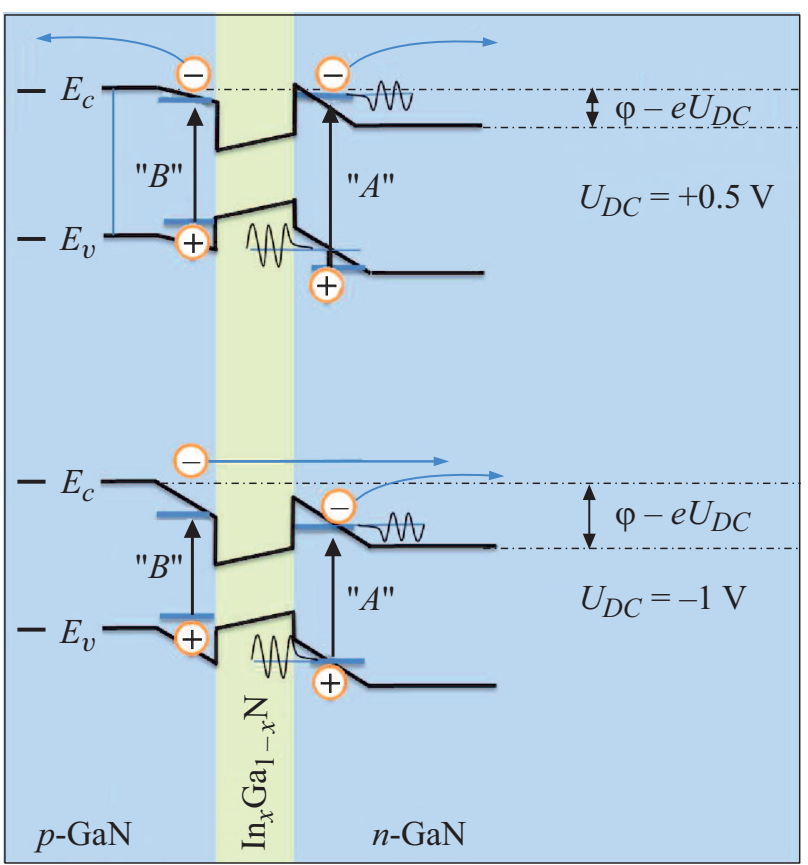

Рис. 3. Схема зонной структуры при двух смещениях $p-n$-перехода $U_{D C}$ для гетероструктуры с одной квантовой ямой.

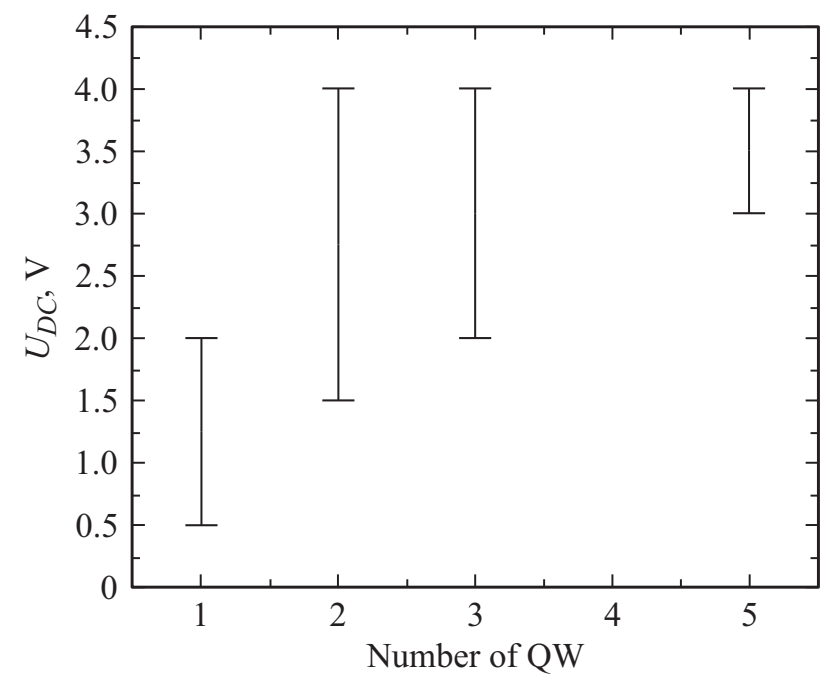

Рис. 4. Диапазон смещений $p-n$-перехода, при котором возникает фотореверсивный эффект, в зависимости от количества квантовых ям в активной области.

Заметим, что указанные энергии связаны только с барьерными слоями. Особенности, соответствующие квантово-размерной области гетероструктуры, находятся в области энергий $\sim 2.5$ эВ и слабо выделяются на фоне поглощения в барьерах.

На рис. 4 показана зависимость диапазона смещений, при которых наблюдается фотореверсивный эффект, от числа КЯ. Наблюдаемый рост среднего значения смещения из этого диапазона связан с тем, что при 
увеличении количества КЯ увеличивается концентрация формирующих обратный ток „барьерных“ электронов, возникающих в нелегированных слоях $\mathrm{GaN}$ между КЯ. Таким образом, с ростом числа КЯ компенсация электронов, образованных в нелегированных барьерах (типа „A“), происходит при больших положительных смещениях, и нижняя граница диапазона смещений на рис. 4 увеличивается.

\section{4. Заключение}

При комнатной температуре зарегистрированы спектры ФТ для разных смещений $p-n$-перехода $U_{D C}$ для образцов с 1, 2, 3 и 5 КЯ в активной области. Обнаружено, что для всех образцов существует определенное смещение, при котором ток, возбуждаемый падающим на структуру излучением, может иметь разное направление в зависимости от длины волны этого излучения. Предположительно, электроны, возбуждаемые в барьерных слоях, создают обратный ток, в то время как электроны, возбуждаемые вблизи активной области с акцепторного уровня в слое $p-\mathrm{GaN}$, создают прямой ток даже при отрицательных смещениях $U_{D C}$. Установлен диапазон смещений $p-n$-перехода, при котором наблюдается указанный эффект для структур с разным количеством квантовых ям в активной области. С ростом количества квантовых ям в активной области эффект реверсивного тока наблюдается при больших смещениях $p$-n-перехода.

\section{Конфликт интересов}

Авторы заявляют, что у них нет конфликта интересов.

\section{Список литературы}

[1] S.M. Sze, K.K. Ng. Physics of Semiconductor Devices (Hoboken, John Wiley \& Sons, Inc., 2006).

[2] E.F. Schubert. Light-Emitting Diodes (Cambridge, Cambridge University Press, 2006).

[3] K. Seeger. Semiconductor Physics (Berlin, Springer Berlin Heidelberg, 1991) v. 40.

[4] A.B.M.H. Islam, J.-I. Shim, D.-S. Shin. IEEE J. Quan. Electron., 55, 1(2019).

[5] A. Bercha, W. Trzeciakowski, M. Gładysiewicz-Kudrawiec, Y. Ivonyak, S. Grzanka. J. Appl. Phys., 125, 115702 (2019).

[6] N. Zhang, Z. Liu, Z. Si, P. Ren, X.-D. Wang, X.-X. Feng, P. Dong, C.-X. Du, S.-X. Zhu, B.-L. Fu, H.-X. Lu, J.-M. Li, J.-X. Wang. Chinese Phys. Lett., 30, 087101 (2013).

[7] S.H. Han, D.Y. Lee, S.J. Lee, C.Y. Cho, M.K. Kwon, S.P. Lee, D.Y. Noh, D.J. Kim, Y.C. Kim, S.J. Park. Appl. Phys. Lett., 94, 1 (2009).

[8] G. Franssen, P. Perlin, T. Suski. Phys. Rev. B, 69, 45310 (2004).

[9] D. Shin, J. Lee, J. Shim. IEEE J. Quan. Electron., 49, 1062 (2013).

[10] S.-I. Park, J.-I. Lee, D.-H. Jang, H.-S. Kim, D.-S. Shin, H.-Y. Ryu, J.-I. Shim. IEEE J. Quant. Electron., 48, 500 (2012).
[11] M. A. Reshchikov, H. Morkoç. J. Appl. Phys., 97, 061301 (2005).

Редактор Г.А. Оганесян

\section{Photoreversible current in InGaN/GaN based LED heterostructures with different number of QWs}

A.E. Aslanyan ${ }^{1}$, L.P. Avakyants ${ }^{1}$, A.V. Chervyakov ${ }^{1}$, A.N. Turkin ${ }^{1}$, V.A. Kureshov ${ }^{2}$, D.R. Sabitov ${ }^{1}$, A.A. Marmalyuk ${ }^{2}$

${ }^{1}$ Lomonosov Moscow State University

(Faculty of Physics), 119991 Moscow, Russia

${ }^{2}$ Sigm Plus Co, 117342 Moscow, Russia

Abstract InGaN/GaN-based LED heterostructures with a different number of quantum wells were studied by photocurrent spectroscopy in the wavelength range of $350-500 \mathrm{~nm}$. As a result of the analysis of a series of spectra obtained at various $p-n$-junction biases, the effect of a change in the direction of the photocurrent with a change in the excitation wavelength (photoreversive effect) was discovered. A range of displacements of the $p-n$-junction is established at which this effect is observed for structures with different numbers of quantum wells in the active region. 